\title{
Preliminary Trajectories in Dietary Behaviors during the COVID-19 Pandemic: A Public Health Call to Action to Face Obesity
}

\author{
Roberta Zupo ${ }^{1, *(1)}$, Fabio Castellana ${ }^{1}$, Rodolfo Sardone ${ }^{1}$, Annamaria Sila ${ }^{1}$, \\ Vito Angelo Giagulli $^{2}\left({ }^{\circledR}\right.$, Vincenzo Triggiani ${ }^{3}{ }^{(0)}$, Raffaele Ivan Cincione ${ }^{4}$, Gianluigi Giannelli ${ }^{5}$ \\ and Giovanni De Pergola ${ }^{1,6}$ \\ 1 Population Health Unit “Salus in Apulia Study"-National Institute of Gastroenterology "Saverio de Bellis", \\ Research Hospital, 70013 Bari, Italy; castellanafabio@hotmail.it (F.C.); rodolfo.sardone@irccsdebellis.it (R.S.); \\ annamaria.sila@irccsdebellis.it (A.S.); gdepergola@libero.it (G.D.P.) \\ 2 Interdisciplinary Department of Medicine-Section of Internal Medicine, Geriatrics, Endocrinology and Rare \\ Diseases, School of Medicine, University of Bari “Aldo Moro", 70121 Bari, Italy; vitogiagulli58@gmail.com \\ 3 Section of Internal Medicine, Geriatrics, Endocrinology and Rare Disease, Interdisciplinary Department of \\ Medicine, School of Medicine, University of Bari, 70121 Bari, Italy; vincenzo.triggiani@uniba.it \\ 4 Department of Clinical and Experimental Medicine, University of Foggia, 71122 Foggia, Italy; \\ ivan.cincione@unifg.it \\ 5 Scientific Direction, National Institute of Gastroenterology "Saverio de Bellis", Research Hospital, \\ 70013 Bari, Italy; gianluigi.giannelli@irccsdebellis.it \\ 6 Clinical Nutrition Unit, Department of Biomedical Science and Human Oncology, University of Bari, \\ School of Medicine, Policlinico, 70121 Bari, Italy \\ * Correspondence: zuporoberta@gmail.com or roberta.zupo@irccsdebellis.it; Tel.: +39-328-295-4840
}

Received: 18 August 2020; Accepted: 24 September 2020; Published: 27 September 2020

\begin{abstract}
The world is currently struggling to face the coronavirus pandemic (COVID-19), and many countries have imposed lockdowns and recommended quarantine to limit both the spread of the virus and overwhelming demands for medical care. Direct implications include the disruption of work routines, boredom, depression, increased calorie consumption, and other similar harmful effects. The present narrative review article briefly analyzes the preliminary effects of the quarantine lifestyle from the standpoint of dietary habits. In six different databases, we searched for original articles up to 10 August 2020, assessing eating habits among populations during the COVID-19 pandemic, and recorded any change in the intake of major food categories, as well as changes in body weight. The research strategy yielded 364 articles, from which we selected 12 articles that fitted our goal. Our preliminary findings revealed a sharp rise of carbohydrates sources consumption, especially those with a high glycemic index (i.e., homemade pizza, bread, cake, and pastries), as well as more frequent snacks. A high consumption of fruits and vegetables, and protein sources, particularly pulses, was also recorded, although there was no clear peak of increase in the latter. Data concerning the consumption of junk foods lacked consistency, while there was a decreased alcohol intake and fresh fish/seafood consumption. As a possible connection, people gained body weight. Therefore, in the realistic perspective of a continuing global health emergency situation, timely preventive measures are needed to counteract obesity-related behaviors in the long-term, so as to prevent further health complications.
\end{abstract}

Keywords: COVID-19; diet; dietary changes; body weight; obesity 


\section{Introduction}

Globally, as of 19 July 2020, there were 14,043,176 confirmed cases of Coronavirus Disease (COVID-19) reported to the World Health Organization (WHO), including 597,583 deaths [1]. Many countries imposed a stringent lockdown in order to enforce social distancing and prevent the spread of infection [2]. The use of social distancing, face masks, gloves, and other individual protection measures is having a massive impact on reducing the current peak of active cases but, as recently demonstrated, over time, a decreasing sense of alarm about the pandemic may contribute to a new, larger second wave of epidemics [3]. In any case, the enforced confinement has upset our life priorities and changed the way we live in several ways, ranging from working behaviors (e.g., smart working, job cuts) to the psychological field, fostering the onset of depression, boredom, sedentary activities, and several more harmful effects on life habits [4,5]. Moreover, constantly hearing or reading about the pandemic during quarantine without a break has stressfully affected most people. Stress and boredom are both drivers of overeating, as people resort to sugary "comfort foods," resulting in the introduction of more energy/calories and an increased craving for food [6]. The latter is a kind of feeling-state driven by emotional (intense desire to eat), behavioral (seeking food), cognitive (thoughts about food), and physiological (salivation) sensations [7]. In this context, the introduction of carbohydrate-rich foods in particular may be an unconscious form of self-medication against adverse stimuli, stimulating serotonin release, which positively affects the mood. Interestingly, the effect of carbohydrate craving in increasingly low moods is proportional to the food glycemic index. The closely involved downside is that these unhealthy dietary behaviors promote the development of obesity, inducing a chronic systemic inflammatory status that, concurrently with other chronic non-communicable conditions such as dyslipidemia, hypertension, heart diseases, diabetes, and lung disease, may increase the risk of more severe complications due to COVID-19 [8]. In this regard, a recent Italian snapshot showed that patients with excess weight admitted to a medical ward for COVID-19-related pneumonia, even younger ones, more frequently needed assisted ventilation and access to intensive or semi-intensive care units than normal weight patients [9]. Sleep disorders also contribute to worsen the quarantine-related scenario, further worsening stress and increasing food intake, thus giving rise to a dangerous vicious cycle [10].

From the perspective of the current stress-overeating situation, nutritional approaches have become a primary goal to counteract the onset of risky dietary behaviors. In this light, we conducted this narrative synthesis with the aim of identifying a preliminary trend of dietary changes during the period of lockdown, also highlighting some findings concerning weight changes, so as to stimulate a call to action to combat the obesity issue within the pandemic. This preliminary synthesis, however, also hopes to stimulate research aimed at defining the risk of COVID-19 infection and the consequent mortality of people affected by obesity, as well as providing food for thought to better orientate risk management, both remotely (e.g., by investing in training healthcare professionals dedicated to nutritional counseling) and in common clinical practice.

\section{Methods}

The present was a narrative review article. We performed separate searches in the US National Library of Medicine (PubMed, Bethesda, MD 20894, USA), Medical Literature Analysis and Retrieval System Online (MEDLINE), EMBASE, Scopus, Ovid, and Google Scholar databases to find original articles on dietary changes and behaviors during the COVID-19 pandemic. The search strategy used in PubMed and MEDLINE and adapted to the other four electronic sources is shown in Table 1. A PRISMA flow diagram of the search procedure is also provided (see Figure 1). The references of the included studies were analyzed for additional references of interest. The last search for the purposes of the present narrative review was performed on 10 August 2020. No language restriction was adopted. Given the novelty of the topic, and therefore small number of data, no skimming was applied to the study design, and both longitudinal and cross-sectional observations were included. For the same reason, no age range was applied to the selected study populations. Two investigators (R.Z., F.C.) 
independently, and in duplicate, searched for papers, screened titles and abstracts of the retrieved articles, reviewed the full-texts, and selected articles for inclusion. The following inclusion criteria were applied: original studies assessing dietary habits among populations during the COVID-19 pandemic with no discrimination between methods of administration (online questionnaire or phone interview) or for estimating intake (daily or weekly frequency). Technical reports, letters to the editor, and systematic and narrative review articles were excluded.

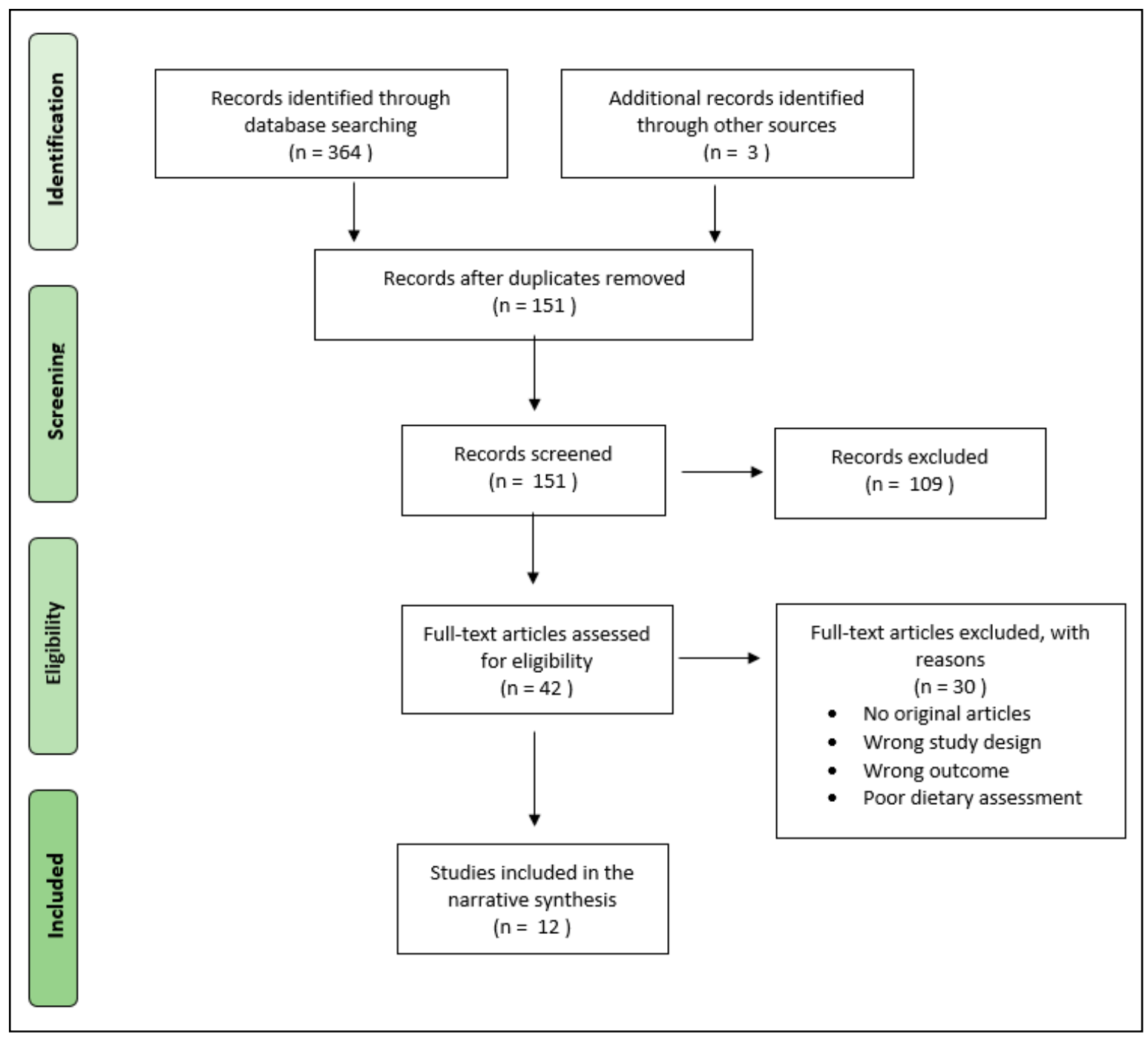

Figure 1. Preferred Reporting Items for Systematic reviews and Meta-Analyses (PRISMA) flow diagram of search procedure. 
Table 1. Search strategy used in the US National Library of Medicine (PubMed) and Medical Literature Analysis and Retrieval System Online (MEDLINE), according to the selected descriptors.

\begin{tabular}{cc}
\hline Strategy & Descriptors Used \\
\hline \#1 & $\begin{array}{c}\text { (diet[tiab]) OR (feeding[tiab])) OR (habits[tiab]) OR (dietary lifestyle[tiab]) OR (dietary } \\
\text { habits[tiab]) OR (dietary [tiab]) OR (dietary pattern[tiab]) OR (dietary behavior[tiab]) OR } \\
\text { (food[tiab]) OR (foods[tiab]) OR (food habits[tiab]) OR (nutritional habits[tiab]) OR (eating } \\
\text { habits[tiab]) OR (eating[tiab]) }\end{array}$ \\
\hline \#2 & $\begin{array}{c}\text { (change[tiab]) OR (changes[tiab]) OR (modifications[tiab]) OR (alterations[tiab]) OR } \\
\text { (alteration[tiab]) OR (different[tiab]) OR (differences [tiab]) }\end{array}$ \\
\hline$\# 3$ & $\begin{array}{c}\text { (sars cov 2[tiab]) OR (covid 19[tiab]) OR (severe acute respiratory syndrome coronavirus } \\
\text { 2[tiab]) }\end{array}$ \\
\hline 4 & \# 1 AND \# 2 AND \# 3
\end{tabular}

The following information was extracted independently and in duplicate by the same investigators in a piloted form: (1) general information in the selected studies (study design, sample size, country, methods for assessing diet, and estimating intake); (2) items derived from the nutritional survey (changes in body weight, carbohydrate intake, junk foods, dressing fats, protein sources, snacks, as well as alcohol intake, and also information about home-cooking practice). The data on carbohydrate consumption were divided by key categories into four subgroups according to major categories (sugary foods, fruit, vegetables, and cereals). Likewise, we sorted the "weight changes" item into "gain," "loss," and "none" based on whether any difference in body weight occurred during the lockdown period. Consistency in the reported items for the assessment of dietary habits among selected studies was verified. Data were cross-checked, any discrepancy was discussed, and disagreements were resolved by a senior researcher (RS).

\section{Results}

\subsection{Overview of Selected Studies}

In total, 12 studies were identified for the purpose of this narrative review. Table 2 summarizes descriptive characteristics of the included studies. The longitudinal setting characterizing a third (four out of 12) of the selected studies allows them to be accurately compared before and during the COVID-19 dietary settings. Meanwhile, the cross-sectional approach of the remaining studies records offers a snapshot of the dietary settings and may possibly strengthen the longitudinal observations. Daily or weekly frequency of foods consumption was adopted to assess changes in foods intake, using phone interviews or online questionnaires. Carbohydrate sources (sugary foods, fruit, vegetables and cereals), fast foods, dressing fats, protein sources (pulses, meat, dairy products, fish), snacks, and alcoholic beverages consumption were evaluated as major food items. Some surveys showed a slight lack of completeness, due to the heterogeneous nature of the investigations. In addition, we observed that half of the adopted study surveys reported on the practice of cooking meals at home. Seven studies reported about changes in body weight, as "gain," "loss," or "no change." Tables 3 and 4 summarize the dietary characteristics of the study findings, subdivided by principal foods intake, and including body weight changes, home-cooking practice, and overall findings. Figure 2 represents food trajectories triggered by the COVID-19 home confinement. Lastly, some information about reported changes in physical activity level is provided in Table 5. 
Table 2. Descriptive characteristics of included studies.

\begin{tabular}{|c|c|c|c|c|c|c|c|}
\hline \multicolumn{8}{|c|}{ Study Details } \\
\hline Ref. & Authors, Year & Sample Size & Study Group & Study Design & Country & $\begin{array}{c}\text { Method of } \\
\text { Estimating Intake }\end{array}$ & $\begin{array}{l}\text { Dietary Assessment } \\
\text { Method }\end{array}$ \\
\hline [11] & Sidor et al., 2020 & 1097 & $18-71$ years & Cross-sectional & Poland & $\begin{array}{l}\text { Daily/weekly } \\
\text { frequency }\end{array}$ & Online questionnaire \\
\hline [12] & Górnicka et al., 2020 & 2.381 & $18+$ years & Cross-sectional & Poland & $\begin{array}{l}\text { Daily/weekly } \\
\text { frequency }\end{array}$ & Online questionnaire \\
\hline [13] & Ghosh et al., 2020 & 150 & $\begin{array}{l}\text { Middle- } \\
\text { aged adults }\end{array}$ & Longitudinal & India & Daily frequency & Phone interview \\
\hline [14] & Di Renzo et al., 2020 & 3533 & $12-86$ years & Cross-sectional & Italy & $\begin{array}{l}\text { Daily/weekly } \\
\text { frequency }\end{array}$ & $\begin{array}{l}\text { Online questionnaire } \\
\text { Eating Habits and } \\
\text { Lifestyle Changes in } \\
\text { COVID19 lockdown } \\
\text { (EHLC-COVID-19) }\end{array}$ \\
\hline [15] & Romeo-Arroyo et al., 2020 & 600 & $18-68$ years & Cross-sectional & Spain & Weekly frequency & Online questionnaire \\
\hline [16] & Pellegrini et al., 2020 & 150 & $18-75$ years & Cross-sectional & Italy & $\begin{array}{c}\text { Daily/weekly } \\
\text { frequency }\end{array}$ & Online questionnaire \\
\hline [17] & Wang et al., 2020 & 2289 & $18-81$ years & Cross-sectional & China & Weekly frequency & Online questionnaire \\
\hline [18] & Rodríguez-Pérez et al., 2020 & 7514 & $>18$ years & Longitudinal & Spain & $\begin{array}{l}\text { Daily/weekly } \\
\text { frequency }\end{array}$ & Online questionnaire \\
\hline [19] & Pietrobelli et al., 2020 & 41 & $6-18$ years & Longitudinal & Italy & Daily frequency & Phone interview \\
\hline [20] & Reyes-Olavarría et al., 2020 & 700 & $18-62$ years & Cross-sectional & Chile & $\begin{array}{l}\text { Daily/weekly } \\
\text { frequency }\end{array}$ & Online questionnaire \\
\hline [21] & Ruiz-Roso et al., 2020 & 820 & 10-19 years & Longitudinal & $\begin{array}{l}\text { Italy, Spain, Chile, } \\
\text { Colombia, Brazil }\end{array}$ & Weekly frequency & $\begin{array}{l}\text { Online questionnaire } \\
\text { (National School Health } \\
\text { Survey-PeNSE survey) }\end{array}$ \\
\hline [22] & Scarmozzino et al., 2020 & 1.929 & - & Cross-sectional & Italy & Weekly frequency & Online questionnaire \\
\hline
\end{tabular}


Table 3. Dietary characteristics of the study findings by principal carbohydrate sources (sugary foods, fruit, vegetables, and cereals), body weight changes (gain, loss, none) and overall findings.

\begin{tabular}{|c|c|c|c|c|c|c|c|c|}
\hline \multirow{2}{*}{ Authors, Year [Ref.] } & \multicolumn{3}{|c|}{ Body Weight Changes } & \multicolumn{4}{|c|}{ Carbohydrate Sources } & \multirow{2}{*}{ Overall Findings } \\
\hline & Gain & Loss & None & Sugary Food & Fruit & Vegetables & Cereals & \\
\hline Ghosh et al., 2020 [13] & $19 \%$ & $33 \%$ & $48 \%$ & $\begin{array}{c}7 \% \text { reported } 25-50 \% \\
\text { more sugar intake }\end{array}$ & $\begin{array}{l}20 \% \text { reported } 25-50 \% \\
\text { more fruits intake }\end{array}$ & $\begin{array}{c}9 \% \text { increased } \\
\text { servings/day ( } 3 \text { or } \\
\text { more) }\end{array}$ & $\begin{array}{l}21 \% \text { increased } \\
\text { cereals intake } \\
\text { (rice, grains) }\end{array}$ & $\begin{array}{l}\text { Increased carbohydrate, } \\
\text { snacking and fruit intake, } \\
\text { and home cooked meals }\end{array}$ \\
\hline Ruiz-Roso et al., 2020 [21] & & - & & $\begin{array}{c}47.4 \% \text { increased sweet } \\
\text { foods intake }(>4 / \text { week }) \\
\text { versus } 40.6 \% \\
\text { pre-COVID-19. Ditto } \\
\text { for sugar beverages } \\
(23.8 \% \text { against } 22.7 \%)\end{array}$ & $\begin{array}{c}58.6 \% \text { increase } \\
\text { (>4/week) versus } 53.9 \% \\
\text { pre-COVID-19 }\end{array}$ & $\begin{array}{c}70.8 \% \text { increase } \\
(>4 / \text { week) versus } 66.2 \% \\
\text { pre-COVID-19 }\end{array}$ & - & $\begin{array}{l}\text { Increased pulses, fruit, and } \\
\text { vegetables intake, and } \\
\text { home cooked meals. } \\
\text { Higher sweet food intake. } \\
\text { The overall diet quality did } \\
\text { not improve. }\end{array}$ \\
\hline $\begin{array}{c}\text { Rodríguez-Pérez et al., } \\
2020[18]\end{array}$ & $12 \%$ & - & $47 \%$ & $\begin{array}{c}\text { Up to } 21 \% \text { decreased } \\
\text { daily sweet beverages } \\
\text { intake }\end{array}$ & $\begin{array}{l}\text { Up to } 18 \% \text { increased } \\
\text { daily intake }\end{array}$ & $\begin{array}{l}\text { Up to } 19 \% \text { increased } \\
\text { daily intake }\end{array}$ & - & $\begin{array}{l}\text { Higher intake of fruits, } \\
\text { vegetables, and pulses and } \\
\text { lower intake of red meat, } \\
\text { alcohol, and fried foods. }\end{array}$ \\
\hline Pietrobelli et al., 2020 [19] & & - & & $\begin{array}{c}\text { Increase of sugary } \\
\text { drinks }(0.40 \pm 0.90 \text { to } \\
0.90 \pm 1.16 \\
\text { servings/day })\end{array}$ & $\begin{array}{c}\text { Increased }(1.16 \pm 0.74 \text { to } \\
1.39 \pm 0.70 \\
\text { servings/day })\end{array}$ & $\begin{array}{c}\text { Increased }(1.34 \pm 0.74 \text { to } \\
1.27 \pm 0.69 \\
\text { servings/day })\end{array}$ & - & $\begin{array}{l}\text { No changes in reported } \\
\text { vegetables intake. Fruit } \\
\text { intake increased. Potato } \\
\text { chip, red meat, and sugary } \\
\text { drink intakes increased } \\
\text { significantly. }\end{array}$ \\
\hline Sidor et al., 2020 [11] & $29.9 \%$ & $18.6 \%$ & - & $\begin{array}{l}\text { One-third consumed at } \\
\text { least once or more/day }\end{array}$ & $\begin{array}{c}\text { One-third did not } \\
\text { consume fresh } \\
\text { vegetables and fruits on } \\
\text { a daily basis }\end{array}$ & $\begin{array}{c}\text { One-third did not } \\
\text { consume fresh } \\
\text { vegetables and fruits on } \\
\text { a daily basis }\end{array}$ & $\begin{array}{l}\text { The majority } \\
(64.2 \%) \text { consumed } \\
\text { grains once or } \\
\text { more/day }\end{array}$ & $\begin{array}{l}\text { One-third of people } \\
\text { surveyed did not consume } \\
\text { fresh vegetables and fruits } \\
\text { on a daily basis, while the } \\
\text { same proportion admitted } \\
\text { to consuming sweets at } \\
\text { least once every day. Obese } \\
\text { people surveyed tended to } \\
\text { eat vegetables, fruits, and } \\
\text { pulses less frequently, and } \\
\text { salty foods, meat, and dairy } \\
\text { more often. }\end{array}$ \\
\hline
\end{tabular}


Table 3. Cont

\begin{tabular}{|c|c|c|c|c|c|c|c|c|}
\hline \multirow{2}{*}{ Authors, Year [Ref.] } & \multicolumn{3}{|c|}{ Body Weight Changes } & \multicolumn{4}{|c|}{ Carbohydrate Sources } & \multirow{2}{*}{ Overall Findings } \\
\hline & Gain & Loss & None & Sugary Food & Fruit & Vegetables & Cereals & \\
\hline Di Renzo et al., 2020 [14] & $48.6 \%$ & $13.9 \%$ & $37.4 \%$ & $\begin{array}{c}43 \% \text { increased } \\
\text { homemade sweets }\end{array}$ & $37.4 \%$ increase & $37.4 \%$ increase & $\begin{array}{l}\text { Up to } 40 \% \text { increased } \\
\text { (homemade pizza, } \\
\text { fresh bread, cereals) }\end{array}$ & $\begin{array}{l}\text { Increased homemade foods } \\
\text { (e.g., sweets, pizza and } \\
\text { bread), cereals, and pulses, } \\
\text { and decreased fresh fish, } \\
\text { packaged sweets and } \\
\text { baked products, delivery } \\
\text { foods and alcohol intake }\end{array}$ \\
\hline $\begin{array}{c}\text { Romeo-Arroyo et al., } \\
2020 \text { [15] }\end{array}$ & & - & & $\begin{array}{l}\text { Over } 50 \% \text { increased } \\
\text { sweets intake }\end{array}$ & $35 \%$ increase & $30 \%$ increase & $\begin{array}{l}\text { Increase of cereals } \\
(20 \%) \text {, pasta/rice } \\
(39 \%) \text {, and bread } \\
(36 \%) \text { consumption }\end{array}$ & $\begin{array}{l}\text { Increased baking, fruits, } \\
\text { and vegetables intake }\end{array}$ \\
\hline $\begin{array}{c}\text { Scarmozzino et al., } 2020 \\
\text { [22] }\end{array}$ & $19.5 \%$ & - & $50.7 \%$ & $\begin{array}{c}42.5 \% \text { increased } \\
\text { chocolate, cakes, and } \\
\text { ice creams }\end{array}$ & $21.2 \%$ increase & $21.2 \%$ increase & - & $\begin{array}{l}\text { Increased consumption of } \\
\text { fresh fruit and vegetables. } \\
\text { Decreased alcohol } \\
\text { consumption }\end{array}$ \\
\hline Górnicka et al., 2020 [12] & & - & & $\begin{array}{c}39.9 \% \text { increased } \\
\text { homemade pastries } \\
\text { intake, } 8.4 \% \text { decreased } \\
\text { sugar-sweetened } \\
\text { beverages, and } 5 \% \\
\text { decreased energy drink } \\
\text { intake }\end{array}$ & $\begin{array}{l}20.1 \% \text { decreased } \\
\text { servings/day } \\
\text { consumption }\end{array}$ & $\begin{array}{c}\text { Almost } 19 \% \text { decreased } \\
\text { servings/day } \\
\text { consumption }\end{array}$ & $\begin{array}{l}16.3 \% \text { increased } \\
\text { whole grain } \\
\text { products intake }\end{array}$ & $\begin{array}{c}\text { Highly increased } \\
\text { consumption of homemade } \\
\text { pastries. Increased } \\
\text { consumption of eggs, } \\
\text { pulses, and cereals, as well } \\
\text { as alcohol. Decreased fish, } \\
\text { fruit, and vegetable intake. }\end{array}$ \\
\hline $\begin{array}{l}\text { Reyes-Olavarría et al., } \\
2020[20]\end{array}$ & $32 \%$ & $17 \%$ & $51 \%$ & - & $\begin{array}{l}30.9 \% \text { increased daily } \\
\text { intake }\end{array}$ & $\begin{array}{l}30.9 \% \text { Increased daily } \\
\text { intake }\end{array}$ & - & $\begin{array}{l}\text { Increased fruit and } \\
\text { vegetables consumption, } \\
\text { and home cooked meals. } \\
\text { Higher junk and fried foods } \\
\text { intake. The overall diet } \\
\text { quality did not improve. }\end{array}$ \\
\hline Pellegrini et al., 2020 [16] & $\begin{array}{r}\text { Self-re } \\
\mathrm{BN} \\
\text { incre }\end{array}$ & $\begin{array}{l}\text { orted we } \\
\text { signific } \\
\text { sed by } 1\end{array}$ & $\begin{array}{l}\text { ght and } \\
\text { htly } \\
51 \mathrm{~kg}\end{array}$ & $\begin{array}{l}72 \% \text { reported equal or } \\
\text { greater sweets } \\
\text { consumption than } \\
\text { pre-COVID-19 }\end{array}$ & $\begin{array}{l}81 \% \text { reported equal or } \\
\text { greater consumption } \\
\text { than pre-COVID-19 }\end{array}$ & $\begin{array}{l}81 \% \text { reported equal or } \\
\text { greater consumption of } \\
\text { cereals than } \\
\text { pre-COVID-19 }\end{array}$ & $\begin{array}{r}\text { Increased freque } \\
\text { More sweets ar }\end{array}$ & $\begin{array}{l}\text { acy of overall food intake. } \\
\text { d snacks consumption. }\end{array}$ \\
\hline Wang et al., 2020 [17] & & & 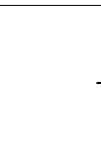 & & $\begin{array}{c}30 \% \text { reported } \\
\text { consuming more } \\
\text { vegetables and fruit, } \\
\text { especially women }\end{array}$ & $\begin{array}{c}\text { Increased consumption } \\
(250-400 \mathrm{~g} / \text { day }) \\
\text { especially men }\end{array}$ & $\begin{array}{l}\text { Higher intake of fr } \\
\text { Increased }\end{array}$ & $\begin{array}{l}\text { uits, vegetables, and cereals. } \\
\text { nacking frequency. }\end{array}$ \\
\hline
\end{tabular}


Table 4. Dietary characteristics of the study findings by other major principal food sources. Data on home-cooking habits were also included.

\begin{tabular}{|c|c|c|c|c|c|c|}
\hline Authors, Year [Ref.] & Junk/Fast Foods & Dressing Fat & $\begin{array}{l}\text { Protein } \\
\text { Sources }\end{array}$ & Snacks & Alcohol & $\begin{array}{c}\text { Home } \\
\text { Cooking }\end{array}$ \\
\hline Ghosh et al., 2020 [13] & - & $\begin{array}{c}5 \% \text { increased overall } \\
\text { fat intake (ghee, } \\
\text { butter) }\end{array}$ & $\begin{array}{l}\text { 3\% increased overall protein intake } \\
\text { (eggs, fish, meat, pulses, soybean) }\end{array}$ & $\begin{array}{l}23 \% \text { increase snacking } \\
\text { frequency }(>4 / \text { day })\end{array}$ & $\begin{array}{c}8 \% \text { decreased daily } \\
\text { intake by } 25-50 \% \\
\text { versus } 3 \% \text { of increase }\end{array}$ & Widespread (97\%) \\
\hline Ruiz-Roso et al., 2020 [21] & $\begin{array}{l}\text { Up to } 18.5 \% \text { increase } \\
(>4 / \text { week })\end{array}$ & - & $\begin{array}{l}23.6 \% \text { increased pulses intake } \\
(>4 / \text { week) against } 22.8 \% \text { before }\end{array}$ & & - & \\
\hline Rodríguez-Pérez et al., 2020 [18] & $\begin{array}{l}\text { Up to } 34 \% \text { lower fast } \\
\text { foods and processed meat } \\
\text { weekly intake }\end{array}$ & $\begin{array}{l}4 \% \text { increase of olive } \\
\text { oil daily intake }\end{array}$ & $\begin{array}{l}\text { Up to } 75 \% \text { and } 7 \% \text { increase in weekly } \\
\text { fish and pulses intake. Almost } 2 \% \\
\text { decreased daily meat intake. }\end{array}$ & $\begin{array}{l}37.6 \% \text { increased } \\
\text { snacking frequency }\end{array}$ & $\begin{array}{l}57.3 \% \text { decreased } \\
\text { weekly intake. }\end{array}$ & $45.7 \%$ increase \\
\hline Pietrobelli et al., 2020 [19] & $\begin{array}{l}\text { Increased potato chips } \\
\text { intake }(0.07 \pm 0.24 \text { to } 0.61 \\
\pm 0.83 \text { serving/day })\end{array}$ & - & $\begin{array}{c}\text { Increased daily red meat } \\
\text { consumption (from } 1.80 \pm 1.53 \text { to } \\
3.46 \pm 2.45 \text { ) }\end{array}$ & & - & \\
\hline Sidor et al., 2020 [11] & Higher frequency & - & $\begin{array}{l}\text { Dairy, meat, and pulses were } \\
\text { consumed by the majority quite } \\
\text { often during the week }\end{array}$ & Up to $60 \%$ increase & $14.6 \%$ increase & $62.3 \%$ increase \\
\hline Di Renzo et al., 2020 [14] & $29.8 \%$ decrease & - & $\begin{array}{l}\text { Up to } 15 \% \text { increased eggs, meat, and } \\
\text { pulses. Decreased fresh fish } \\
\text { consumption - } 22 \%\end{array}$ & - & $\begin{array}{l}13 \% \text { decreased wine } \\
\text { and beer } \\
\text { consumption }\end{array}$ & - \\
\hline Romeo-Arroyo et al., 2020 [15] & - & & $\begin{array}{l}30 \% \text { increase of eggs, } 39 \% \text { increase of } \\
\text { milk and dairy products, and } 33 \% \\
\text { decrease of fish intake }\end{array}$ & - & $\begin{array}{l}27 \% \text { decreased } \\
\text { alcoholic beverages }\end{array}$ & $\begin{array}{c}\text { Widespread (between } \\
4 \text { and } 6 \text { on a 7-point } \\
\text { scale of agreement) }\end{array}$ \\
\hline Scarmozzino et al., 2020 [22] & & - & & $\begin{array}{l}23.5 \% \text { increased } \\
\text { frequency }\end{array}$ & $\begin{array}{l}36.8 \% \text { decreased wine, } \\
\text { beer, and liquors } \\
\text { consumption }\end{array}$ & - \\
\hline Górnicka et al., 2020 [12] & $36.6 \%$ decrease & - & $\begin{array}{l}\text { Increased low-fat meat and/or eggs } \\
(15.7 \%), \text { pulses }(13.9 \%) \text {, and milk } \\
\text { and dairy products }(20.8 \%) \text { intake. } \\
\text { Decreased fish and seafood (17\%), } \\
\text { and processed meat }(17.7 \%) \text { intake. }\end{array}$ & $\begin{array}{l}19.7 \% \text { decreased salty } \\
\text { snacks }\end{array}$ & $18.1 \%$ increase & $48 \%$ increase \\
\hline Reyes-Olavarría et al., 2020 [20] & $\begin{array}{l}\text { Increased junk and fried } \\
\text { foods intake (1-2 } \\
\text { times/week) }\end{array}$ & - & $\begin{array}{l}\text { About } 25 \% \text { consumed red and white } \\
\text { meat more than } 3 \text { times/week. } 75.1 \% \\
\text { and } 83.7 \% \text { consumed fish and pulses } \\
1-2 \text { times/week. }\end{array}$ & - & $\begin{array}{l}30 \% \text { declared a daily } \\
\text { consumption of } \\
\text { alcohol }\end{array}$ & $59.6 \%$ increase \\
\hline Pellegrini et al., 2020 [16] & - & & $\begin{array}{l}81 \% \text { reported equal or greater } \\
\text { consumption of overall protein } \\
\text { sources than pre-COVID-19 }\end{array}$ & $\begin{array}{l}60.6 \% \text { reported equal } \\
\text { or greater snacking } \\
\text { frequency }\end{array}$ & & - \\
\hline Wang et al., 2020 [17] & - & & $\begin{array}{l}\text { Meats, dairy products, and eggs } \\
\text { fulfilled the recommendation of the } \\
\text { dietary guidelines for Chinese } \\
\text { residents }\end{array}$ & $\begin{array}{c}\text { About } 30 \% \text { reported } \\
\text { an increased snacking } \\
\text { frequency }\end{array}$ & & - \\
\hline
\end{tabular}


Table 5. Details about reported trends on changes in physical activity level during the COVID-19 lockdown.

\begin{tabular}{|c|c|c|c|}
\hline Ref. & Authors, Year & Physical Activity Level & Study Design \\
\hline [11] & Sidor et al., 2020 & - & Cross-sectional \\
\hline [12] & Górnicka et al., 2020 & $43 \%$ decreased & Cross-sectional \\
\hline [13] & Ghosh et al., 2020 & $42 \%$ decreased & Longitudinal \\
\hline [14] & Di Renzo et al., 2020 & $\begin{array}{l}\text { No significant difference between the percentage of people } \\
\text { that did not train before }(37.7 \%) \text { or during }(37.4 \%) \text { the } \\
\text { COVID-19 lockdown. However, a higher frequency of } \\
\text { training during the emergency was found when compared } \\
\text { to the previous period. }\end{array}$ & Cross-sectional \\
\hline [15] & Romeo-Arroyo et al., 2020 & - & Cross-sectional \\
\hline [16] & Pellegrini et al., 2020 & $\begin{array}{l}79.3 \% \text { did not do any physical activity or reduced their } \\
\text { physical activity level compared to pre-COVID-19 }\end{array}$ & Cross-sectional \\
\hline [17] & Wang et al., 2020 & More than $50 \%$ decreased & Cross-sectional \\
\hline [18] & Rodríguez-Pérez et al., 2020 & $59.6 \%$ decreased & Longitudinal \\
\hline [19] & Pietrobelli et al., 2020 & Sports time decreased significantly by $2.30 \pm 4.60 \mathrm{~h} /$ week & Longitudinal \\
\hline [20] & Reyes-Olavarría et al., 2020 & $\begin{array}{c}\text { The highest percentage of subjects passed } \geq 6 \mathrm{~h} \text { sitting or } \\
\text { sedentary activities }(54.4 \%)\end{array}$ & Cross-sectional \\
\hline [21] & Ruiz-Roso et al., 2020 & - & Longitudinal \\
\hline [22] & Scarmozzino et al., 2020 & - & Cross-sectional \\
\hline
\end{tabular}




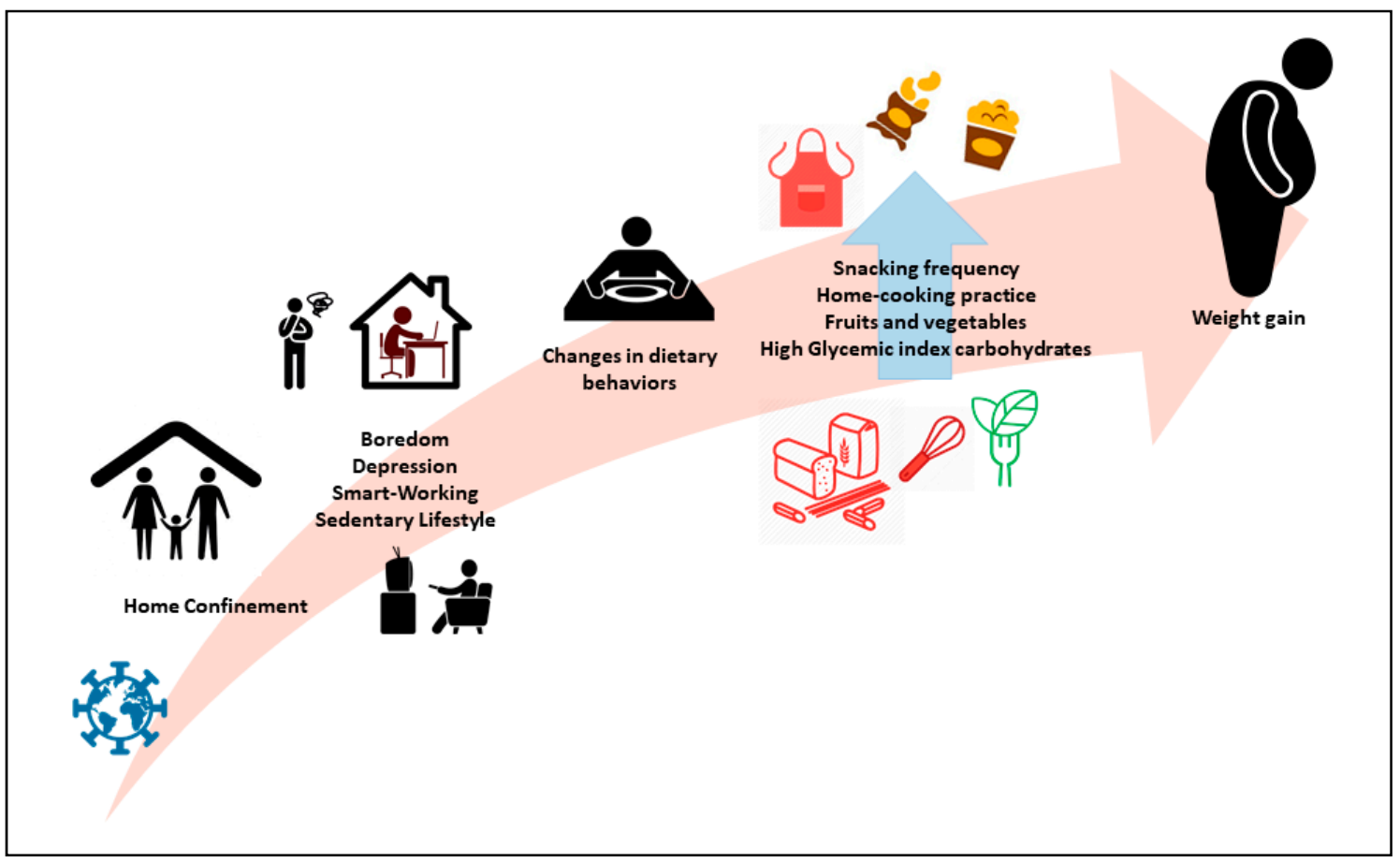

Figure 2. Food trajectories triggered by the COVID-19 home confinement.

\subsection{Single Foods or Food Groups}

Both longitudinal and cross-sectional observations revealed an overall increased consumption of carbohydrates sources. Starting from sugary foods, such as sugary drinks, ice creams, and homemade or packaged sweet foods (i.e., cakes, biscuits, chocolate), we observed a rise in daily/weekly consumption by up to $70 \%$. The same increase resulted for fruit and vegetables consumption, except for the Polish population reported both by Sidor and Rzymski [11] and by Górnicka et al. [12]. In the first case, nearly one-third of the 1097 Polish citizens surveyed did not consume fresh vegetables and fruits on a daily basis, while the same proportion admitted to consuming sweets at least once every day. In the second, about $20 \%$ of the 2381 reported a decreased daily consumption. Among studies investigating cereals intake (rice, grains, flours, and derived products such as pizza or bread), an increase in daily/weekly consumption was evident. In this regard, the only longitudinal observation, carried out by Ghosh et al. on a fairly small Indian sample, revealed a $21 \%$ increase in the daily consumption of grains and rice [13]. Similarly, seven cross-sectional observations showed that a large majority consumed higher amounts of cereals [11-17]. In particular, homemade pizza, fresh bread, pasta, and rice were found to rule the trend among the Italian and Spanish surveys performed over a wide range of ages and sample sizes (increases by up to $40 \%$ and $39 \%$, respectively) [14,15]. The large sample of Poles surveyed by Górnicka et al. [12] likewise reported an increase in whole grain products consumption by more than $16 \%$.

Changes in fat consumption were mainly assessed by recording the intake of dressing fats (olive oil, ghee, butter). It should be noted that although none of the cross-sectional studies reported this information, longitudinal observations showed a slight over-consumption, particularly referred to Indian [13] and Spanish populations [18]. In both these cases, the increased daily consumption as compared to the pre- COVID-19 era did not exceed 5\%.

Eleven studies reported major protein sources (fish, meat, eggs, pulses, and dairy products). Some of them reported a low-moderate increase in pulses consumption. Among longitudinal observations, an increased pulses consumption was declared by about $23.6 \%$ of Indians surveyed ( $>4$ times per week) versus $22.8 \%$ before COVID-19, and 7\% of Spaniards (more times/week than before COVID-19). Similarly, cross-sectional observations showed a higher consumption by Polish [11,12] 
and Italian populations [14]. Particularly, Górnicka et al. reported that low-fat meat, eggs, pulses, and dairy products consumption increased up to $20 \%$, while there was a decrease of processed meat consumption (17.7\%) [12]. In line, Di Renzo reported an overall increase up to $15 \%$ of these four protein sources without discriminating between types of meat [14].

By contrast, studies inquiring about fresh fish and seafood consumption reported an overall decrease in weekly frequency intake (by $22 \%$ and $33 \%$ among Italians and Spaniards respectively, and by $17 \%$ among Poles). Information about meat consumption lacked consistency, despite an apparent trend towards a slight increase. In this regard, one of the Italian surveys reported an apparently increased daily red meat consumption (from $1.80 \pm 1.53$ to $3.46 \pm 2.45$ servings/day) in 41 children and adolescents from Verona within a longitudinal setting [19]. A similar cross-sectional observation on a larger sample size, including subjects aged more than 12 years old belonging to every region, reported an increased egg, meat, and pulse consumption by up to 15\% [14]. Similarly, the majority (81\%) of the overweight Italians surveyed by Pellegrino et al. [16] showed equal or greater consumption of overall protein sources than pre-COVID-19. Again, a quarter of the 700 Chilean investigated by Reyes-Olavarría et al. declared that they consumed red and white meat more than 3 times/week [20]. Conversely, the longitudinal Spanish observation registered a decrease of meat daily intake by almost $2 \%[18]$.

As to eggs and dairy products, although this was not sufficiently investigated, the confinement induced a slightly increased consumption among Indian [13], Italian [14], Polish [11,12], and Spanish [15] populations.

\subsection{Junk Foods, Snacks, and Alcoholic Beverages}

There is still no clear trend for junk food intake during the current pandemic. Among longitudinal observations, Ruiz-Roso et al. found an $18.5 \%$ increase in the weekly frequency of junk food consumption, surveying 820 adolescents from Spain, Italy, Brazil, Colombia, and Chile [21]; the same positive direction regarding potato chips intake among Italian adolescents was found by Pietrobelli et al. [19]. In contrast, other studies reported a reversed trend, probably due to the age group investigated. In fact, data from adult Spanish and Italian populations showed an overall decline in the consumption of this food category ( $34 \%$ and $29.8 \%$ less, respectively).

Particularly noteworthy is that confinement encouraged a significant increase in snack consumption apart from the main daily meals, and this finding was demonstrated in all studies published thus far, regardless of the age group surveyed. The number of people who consumed snacks more than 4 times a day increased by $23 \%$ in India [13], while $37.6 \%$ of Spaniards [18] and 30\% of Chinese [17] increased their overall snacking frequency. Instead, the snapshot of Italians and Poles recorded increases in the frequency of consumption by about $60 \%$ [16] and $23 \%$ [22], respectively.

All studies examining the changes in alcoholic beverages consumption concluded that this dropped, except for a Polish survey (showing a $14.6 \%$ and $18.1 \%$ increase). In detail, $8 \%$ of the Indians and $13 \%$ [14] and $36.8 \%$ [22] of the Italians reduced their daily intake by $25-50 \%$. Similarly, the two Spanish surveys reported a $57.3 \%$ decrease in the weekly frequency of consumption in the longitudinal observation by Rodríguez-Pérez et al. [18], and a 27\% decrease in the cross-sectional evaluation carried out by Romeo-Arroyo et al. [15].

\subsection{Body Weight Changes, Physical Activity Level, and Home-Cooking Habits}

Five of the 12 studies did not report any information about changes in body weight within the populations surveyed. However, the available evidence allows us to assume that almost $50 \%$ of people maintained their starting weight, without undergoing any changes. A considerable proportion gained weight: $19 \%$ of Indians [13], 12\% of Spaniards [18], 29.9\% of Poles [11], 32\% of Chileans [20], and $48.6 \%$ [14] and 19.5\% [22] of Italians. The few data available on people who lost weight reported a 33\% loss among Indians [13], 32\% among Chileans [20], 18.6\% among Poles [11], and 3.9\% among Italians [14,22]. 
As predicted due to the enforced confinement, the level of physical activity recorded a drastic drop to $79 \%$ (Table 5), and this finding likely contributed to the weight trajectories.

Home-cooking became much more common, being practiced by $97 \%$ of Indians [13] and the majority of the Spanish population. An ample increase was also registered in Poland $(62.3 \%$ and $48 \%)[11,12]$ and Chile $(59.6 \%)$ [20].

\section{Discussion}

In this narrative synthesis analyzing the latest data on people's food choices during the COVID-19 confinement periods, the main finding was an initial decline in overall dietary lifestyle. Specifically, the consumption of carbohydrates sources, as well as snacking and home-cooking habits, underwent a substantial increase. Instead, a lack of consistency of data concerning the consumption of meat and junk foods was noted, probably due to the paucity of studies and the heterogeneous age range of the populations surveyed, as well as assessment methods. Moreover, these preliminary data showed a slightly increased consumption of pulses, fruit, and vegetables, and a great increase in the habit of cooking meals at home. As a possible connection, people gained some weight, even if almost half of those surveyed did not declare significant fluctuations. The finding that, as a result of the enforced confinement, people had more desire to cook, and above all to knead, is of particular concern. Thus, the consumption of homemade desserts, bread and pizza, as well as sweet pastries (biscuits, cakes, and other bakery) rapidly rose, inducing a higher exposure to unhealthy foods with a high glycemic index [23]. In other words, these unhealthy dietary habits facilitate a poor control of the body weight, leading to obesity. This is undoubtedly a spontaneous consequence of stress and/or boredom and/or other kinds of negative emotions (so-called "emotional eating"), as opposed to a more limited level of physical activity closely related to the confinement. Moreover, people tended to eat snacks more frequently, and foods outside meals in general, even if they were not really hungry, just as a way to kill boredom. Another key point is the inability to procure fresh food, as people were forced to stay at home, order takeout food, and prefer long shelf-life foods so as to minimize going out to procure groceries. In fact, the limited access to daily grocery shopping led to a reduced consumption of fresh foods in favor of highly processed ones, such as convenience foods, junk foods, snacks, and ready-to-eat cereals, which tend to be high in fats, sugars, and salt. This inclination was matched by the overall drop in fresh fish and seafood intake. The latter, besides being a noble source of protein that is useful in controlling body weight [24], is a well-known source of omega-3 fatty acids, specifically eicosapentaenoic acid (EPA) and docosahexaenoic acid (DHA), with anti-inflammatory effects that could enhance resistance and the recovery from Severe Acute Respiratory Syndrome CoronaVirus-2 (SARS-CoV-2) [25]. Similarly, fresh vegetables and fruits consumption was no less affected, as reported by the Polish survey. This issue may be extremely worrying considering the great emphasis allocated by the latest findings to vitamins and minerals deficiencies, including beta-carotene, vitamin $\mathrm{C}$, and vitamin $\mathrm{E}$, especially in view of their antioxidant and anti-inflammatory properties. In fact, the inadequate intake of these micronutrients is associated with both obesity and impaired immune responses, thus making people more susceptible to viral infections [26,27].

Preliminary data on meat consumption lack consistency, despite an apparent trend towards a slight increase, while eggs and pulses intake seemed to be mildly increased, perhaps because of their easier availability and longer shelf-life compared to fish or meat. The trajectories for junk foods consumption, apparently increased among younger people, are equally controversial. This practice is likely promoted by the possibility of home delivery of food during this period. Lastly, alcoholic beverages consumption registered a general decrease, possibly due to the lack of "social drinking" (i.e., enjoying drinks with friends at bars) or just of spending time with other people. Noteworthily, since a heavy alcohol intake has been demonstrated to weaken the immune system and thus reduce the ability to cope with infectious diseases [28], this may be positive from the long-term emergency perspective. 


\section{Strengths and Limitations}

It is important to highlight the strengths and limitations of the present review. First of all, the limited number of studies weakens the weight of the findings. Secondly, for the same reason, we included two different types of settings, without any skimming. Thirdly, the studies featured different and mostly poorly accurate dietary assessment approaches; indeed, quantification in grams was uncommon. However, this is the first review paper to describe preliminary dietary modifications during the COVID-19 pandemic, and can thus offer early food for thought on this matter.

\section{Conclusions}

Summing up these preliminary data, it is not surprising that a fair percentage of people experienced an increase in body weight. This may pose a severe problem in the highly realistic perspective of a continuing global health emergency situation. Nutrition has become a priority right now, because a well-balanced diet is the best way to get all the essential nutrients we need to ensure normal immune function, while reducing the risk of obesity. More research is urgently needed to better understand the global dietary trend during the COVID-19 emergency in the long term. However, according to these initial data, preventive measures must already be put in place in order to implement basic tools against obesogenic lifestyles within the COVID-19 era. Actions to address the growing challenge of obesity require systematic and multi-sector policy actions, as well as recognition that governments need to address the roots of obesity. A starting point would be to implement comprehensive policies and actions to improve the food environment, for example through restrictions on marketing and taxes. Concurrently, it would be advisable to support research defining the risk of contracting the COVID-19 infection and subsequent mortality in people living with obesity, in addition to providing clear guidance on managing risk factors. Additionally, it would be desirable to provide specific training for health professionals to enable them to support people living with obesity in the COVID-19 era, so as to minimize related risks and unconscious prejudices, as well as to prevent stigma.

Author Contributions: Conceptualization, R.S.; methodology, R.S., R.Z. and F.C.; validation, A.S. and G.G.; investigation, R.Z. and F.C.; writing — original draft preparation, R.Z., F.C. and G.D.P.; writing-review and editing, A.S., V.A.G. and V.T.; visualization, R.I.C., G.D.P. and G.G.; supervision, G.D.P. and R.S. All authors have read and agreed to the published version of the manuscript.

Funding: This research received no external funding.

Conflicts of Interest: The authors declare no conflict of interest.

\section{Abbreviations}

$\begin{array}{ll}\text { COVID-19 } & \text { Coronavirus Disease 19 } \\ \text { WHO } & \text { World Health Organization } \\ \text { MEDLINE } & \text { Medical Literature Analysis and Retrieval System Online } \\ \text { EPA } & \text { Eicosapentaenoic Acid } \\ \text { DHA } & \text { Docosahexaenoic Acid } \\ \text { PRISMA } & \text { Preferred Reporting Items for Systematic Reviews and Meta-Analyses }\end{array}$

\section{References}

1. World Health Organization. Coronavirus Disease (COVID-19) Dashboard. Available online: https: //Covid19.Who.Int/ (accessed on 31 July 2020).

2. Ghosal, S.; Bhattacharyya, R.; Majumder, M. Impact of complete lockdown on total infection and death rates: A hierarchical cluster analysis. Diabetes Metab. Syndr. 2020, 14, 707-711. [CrossRef] [PubMed]

3. López, L.; Rodó, X. The end of social confinement and COVID-19 re-emergence risk. Nat. Hum. Behav. 2020, 4, 746-755. [CrossRef] 
4. Stanton, R.; To, Q.G.; Khalesi, S.; Williams, S.L.; Alley, S.J.; Thwaite, T.L.; Fenning, A.S.; Vandelanotte, C. Depression, Anxiety and Stress during COVID-19: Associations with Changes in Physical Activity, Sleep, Tobacco and Alcohol Use in Australian Adults. Int. J. Environ. Res. Public Health 2020, 17, 4065. [CrossRef] [PubMed]

5. Abbas, A.M.; Kamel, M.M. Dietary habits in adults during quarantine in the context of COVID-19 pandemic. Obes. Med. 2020, 19, 100254. [CrossRef]

6. Moynihan, A.B.; van Tilburg, W.A.P.; Igou, E.R.; Wisman, A.; Donnelly, A.E.; Mulcaire, J.B. Eaten up by boredom: Consuming food to escape awareness of the bored self. Front. Psychol. 2015, 6, 369. [CrossRef]

7. Rodríguez-Martín, B.C.; Meule, A. Food craving: New contributions on its assessment, moderators, and consequences. Front. Psychol. 2015, 6, 21. [CrossRef]

8. Wu, C.; Chen, X.; Cai, Y.; Xia, J.; Zhou, X.; Xu, S.; Huang, H.; Zhang, L.; Zhou, X.; Du, C.; et al. Risk Factors Associated with Acute Respiratory Distress Syndrome and Death in Patients with Coronavirus Disease 2019 Pneumonia in Wuhan, China. JAMA Intern. Med. 2020. [CrossRef]

9. Busetto, L.; Bettini, S.; Fabris, R.; Serra, R.; Dal Pra', C.; Maffei, P.; Rossato, M.; Fioretto, P.; Vettor, R. Obesity and COVID-19: An Italian snapshot. Obesity 2020. [CrossRef]

10. Vgontzas, A.N.; Lin, H.-M.; Papaliaga, M.; Calhoun, S.; Vela-Bueno, A.; Chrousos, G.P.; Bixler, E.O. Short sleep duration and obesity: The role of emotional stress and sleep disturbances. Int. J. Obes. 2008, 32, 801-809. [CrossRef] [PubMed]

11. Sidor, A.; Rzymski, P. Dietary Choices and Habits during COVID-19 Lockdown: Experience from Poland. Nutrients 2020, 12, 1657. [CrossRef] [PubMed]

12. Górnicka, M.; Drywień, M.E.; Zielinska, M.A.; Hamułka, J. Dietary and Lifestyle Changes During COVID-19 and the Subsequent Lockdowns among Polish Adults: A Cross-Sectional Online Survey PLifeCOVID-19 Study. Nutrients 2020, 12, 2324. [CrossRef]

13. Ghosh, A.; Arora, B.; Gupta, R.; Anoop, S.; Misra, A. Effects of nationwide lockdown during COVID-19 epidemic on lifestyle and other medical issues of patients with type 2 diabetes in north India. Diabetes Metab. Syndr. 2020, 14, 917-920. [CrossRef]

14. Di Renzo, L.; Gualtieri, P.; Pivari, F.; Soldati, L.; Attinà, A.; Cinelli, G.; Leggeri, C.; Caparello, G.; Barrea, L.; Scerbo, F.; et al. Eating habits and lifestyle changes during COVID-19 lockdown: An Italian survey. J. Transl. Med. 2020, 18, 229. [CrossRef]

15. Romeo-Arroyo, E.; Mora, M.; Vázquez-Araújo, L. Consumer behavior in confinement times: Food choice and cooking attitudes in Spain. Int. J. Gastron. Food Sci. 2020, 21, 100226. [CrossRef] [PubMed]

16. Pellegrini, M.; Ponzo, V.; Rosato, R.; Scumaci, E.; Goitre, I.; Benso, A.; Belcastro, S.; Crespi, C.; De Michieli, F.; Ghigo, E.; et al. Changes in Weight and Nutritional Habits in Adults with Obesity during the "Lockdown" Period Caused by the COVID-19 Virus Emergency. Nutrients 2020, 12, 2016. [CrossRef] [PubMed]

17. Wang, X.; Lei, S.M.; Le, S.; Yang, Y.; Zhang, B.; Yao, W.; Gao, Z.; Cheng, S. Bidirectional Influence of the COVID-19 Pandemic Lockdowns on Health Behaviors and Quality of Life among Chinese Adults. Int. J. Environ. Res. Public Health 2020, 17, 5575. [CrossRef] [PubMed]

18. Rodríguez-Pérez, C.; Molina-Montes, E.; Verardo, V.; Artacho, R.; García-Villanova, B.; Guerra-Hernández, E.J.; Ruíz-López, M.D. Changes in Dietary Behaviours during the COVID-19 Outbreak Confinement in the Spanish COVIDiet Study. Nutrients 2020, 12, 1730. [CrossRef] [PubMed]

19. Pietrobelli, A.; Pecoraro, L.; Ferruzzi, A.; Heo, M.; Faith, M.; Zoller, T.; Antoniazzi, F.; Piacentini, G.; Fearnbach, S.N.; Heymsfield, S.B. Effects of COVID-19 Lockdown on Lifestyle Behaviors in Children with Obesity Living in Verona, Italy: A Longitudinal Study. Obesity 2020, 28, 1382-1385. [CrossRef] [PubMed]

20. Reyes-Olavarría, D.; Latorre-Román, P.Á.; Guzmán-Guzmán, I.P.; Jerez-Mayorga, D.; Caamaño-Navarrete, F.; Delgado-Floody, P. Positive and Negative Changes in Food Habits, Physical Activity Patterns, and Weight Status during COVID-19 Confinement: Associated Factors in the Chilean Population. Int. J. Environ. Res. Public Health 2020, 17, 5431. [CrossRef]

21. Ruiz-Roso, M.B.; de Carvalho Padilha, P.; Mantilla-Escalante, D.C.; Ulloa, N.; Brun, P.; Acevedo-Correa, D.; Peres, W.A.F.; Martorell, M.; Aires, M.T.; de Oliveira Cardoso, L.; et al. Covid-19 Confinement and Changes of Adolescent's Dietary Trends in Italy, Spain, Chile, Colombia and Brazil. Nutrients 2020, 12, 1807. [CrossRef]

22. Scarmozzino, F.; Visioli, F. Covid-19 and the Subsequent Lockdown Modified Dietary Habits of Almost Half the Population in an Italian Sample. Foods 2020, 9, 675. [CrossRef] [PubMed] 
23. D'Alessandro, A.; De Pergola, G. Mediterranean Diet Pyramid: A Proposal for Italian People. Nutrients 2014, 6, 4302-4316. [CrossRef] [PubMed]

24. Pergola, G.D.; De Pergola, G.; Zupo, R.; Lampignano, L.; Paradiso, S.; Murro, I.; Bartolomeo, N.; Cecere, A.; Ciccone, M.M.; Giannelli, G.; et al. Effects of a Low Carb Diet and Whey Proteins on Anthropometric, Hematochemical and Cardiovascular Parameters in Subjects with Obesity. Endocr. Metab. Immune Disord. Drug Targets 2020, 20. [CrossRef] [PubMed]

25. Das, U.N. Can Bioactive Lipids Inactivate Coronavirus (COVID-19)? Arch. Med. Res. 2020, 51, $282-286$. [CrossRef]

26. Childs, C.E.; Calder, P.C.; Miles, E.A. Diet and Immune Function. Nutrients 2019, 11, 1933. [CrossRef]

27. McAuliffe, S.; Ray, S.; Fallon, E.; Bradfield, J.; Eden, T.; Kohlmeier, M. Dietary micronutrients in the wake of COVID-19: An appraisal of evidence with a focus on high-risk groups and preventative healthcare. BMJ Nutr. Prev. Health 2020. [CrossRef]

28. Simet, S.M.; Sisson, J.H. Alcohol's Effects on Lung Health and Immunity. Alcohol Res. 2015, 37, $199-208$.

(C) 2020 by the authors. Licensee MDPI, Basel, Switzerland. This article is an open access article distributed under the terms and conditions of the Creative Commons Attribution (CC BY) license (http://creativecommons.org/licenses/by/4.0/). 